\title{
Delayed recovery from paralysis by succinylcholine in patient with preoperatively unrecognized and inherited pseudocholinesterase deficiency
}

\author{
Seong-Ho Ok ${ }^{1}$, Min-Kyu Woo ${ }^{2}$, Cheon-Gyu Kim³ ${ }^{3}$ Iljeong Hwang ${ }^{4}$, and Ju-Tae Sohn ${ }^{1}$ \\ ${ }^{1}$ Department of Anesthesiology and Pain Medicine, Institute of Health Sciences, Gyeongsang National University School of Medicine, \\ Gyeongsang National University Hospital, Jinju, Departments of ${ }^{2}$ Anesthesiology and Pain Medicine, ${ }^{3}$ Otorhinolaryngology, Yeil \\ Otorhinolaryngology Clinic, Changwon, ${ }^{4}$ Department of Anesthesiology and Pain Medicine, Gyeongsang National University \\ Hospital, Jinju, Korea
}

Pseudocholinesterase, which is produced by the liver and released into plasma, is involved in the metabolism of muscle relaxants such as succinylcholine and mivacurium, as well as aminoester local anesthetics, including procaine $[1,2]$. Pseudocholinesterase deficiency, arising from inherited, acquired, and iatrogenic causes, produces prolonged neuromuscular blockade after succinylcholine administration used for endotracheal intubation [1]. Here, we report a delayed recovery from paralysis by succinylcholine in a child with genetically inherited pseudocholinesterase deficiency who was not diagnosed preoperatively.

A 7-year-old female patient (body weight $30 \mathrm{~kg}$, height 129 $\mathrm{cm}$ ) was admitted to a private otorhinolaryngology clinic for a tonsillectomy, adenoidectomy, and ventilation tube insertion. The preoperative vital signs and laboratory test findings were within the normal limits. The patient had no specific medication history, and she and all of her family members had no prior anesthetic history of receiving the muscle relaxants succinylcholine or mivacurium. The preoperative physical examination on admission was within normal limits. The patient was premedicated with atropine $(0.01 \mathrm{mg} / \mathrm{kg})$ and dexamethasone $(0.1 \mathrm{mg} / \mathrm{kg})$. Anesthesia was induced with sodium thiopental $(5 \mathrm{mg} / \mathrm{kg})$ and succinylcholine $(1.6 \mathrm{mg} / \mathrm{kg}$ ) while the patient received oxygen $(4 \mathrm{~L} / \mathrm{min})$ through a face mask. Orotracheal intubation was then performed using a reinforced endotracheal tube (internal diameter: 5.5). Then, after we confirmed breath sounds, vecuronium bromide $(0.1 \mathrm{mg} / \mathrm{kg})$ was intravenously administered to maintain muscle relaxation. Anesthesia was maintained with nitrous oxide $(1.5 \mathrm{~L} / \mathrm{min})$ in oxygen $(1.5 \mathrm{~L} / \mathrm{min})$ and sevoflurane (2 vol\%). Intraoperative vital signs were stable. The total anesthesia and operation time was 60 minutes. The operation and anesthesia were uneventful.

Although neuromuscular blockade reversal was attempted with pyridostigmine $(0.3 \mathrm{mg} / \mathrm{kg})$ and glycopyrrolate $(0.007 \mathrm{mg} /$ $\mathrm{kg}$ ), the patient was almost unresponsive to external stimuli, such as endotracheal tube suction, and exhibited apnea. Because our private clinic lacked a peripheral nerve stimulator to assess the extent of neuromuscular blockade and the patient had not regained consciousness 30 minutes after the operation, we suspected that neuromuscular blockade might not have been completely reversed. Thus, we additionally administered intravenous pyridostigmine $(0.17 \mathrm{mg} / \mathrm{kg})$ and glycopyrrolate $(0.003$ $\mathrm{mg} / \mathrm{kg}$ ). Despite this additional administration, the patient was unresponsive and did not regain consciousness. The patient's body temperature was $37.3^{\circ} \mathrm{C}$, and her serum potassium level was within the normal range. Forty minutes after the operation, endotracheal tube suction slightly increased her blood pressure and heart rate, but spontaneous respiration and movement were not observed. Her ventilation was initially controlled and subsequently assisted during apnea with $100 \%$ oxygen via endotracheal tube. Arterial blood gas analysis showed mild respiratory acidosis (pH: 7.22, carbon dioxide partial pressure: $55.6 \mathrm{mmHg}$,

Corresponding author: Ju-Tae Sohn, M.D., Department of Anesthesiology and Pain Medicine, Institute of Health Sciences, Gyeongsang National University School of Medicine, Gyeongsang National University Hospital, 90, Chiram-dong, Jinju 660-702, Korea. Tel: 82-55-750-8586, Fax: 82-55750-8142, E-mail: jtsohn@nongae.gsnu.ac.kr

(c) This is an open-access article distributed under the terms of the Creative Commons Attribution Non-Commercial License (http:// creativecommons.org/licenses/by-nc/3.0/), which permits unrestricted non-commercial use, distribution, and reproduction in any medium, provided the original work is properly cited. 
Table 1. Pseudocholinesterase Level of Patient and Family Members

\begin{tabular}{lccc}
\hline $\begin{array}{c}\text { Family } \\
\text { members }\end{array}$ & $\begin{array}{c}\text { Age } \\
(\mathrm{yr})\end{array}$ & $\begin{array}{c}\text { Pseudocholinesterase } \\
(\mathrm{U} / \mathrm{L})\end{array}$ & $\begin{array}{c}\text { Reference } \\
(\mathrm{U} / \mathrm{L})\end{array}$ \\
\hline Patient & 7 & 213 (postoperative day 2) & $5320-12920$ \\
& & 190 (postoperative day 12) & $5320-12920$ \\
Mother & 42 & 3653 (postoperative day 12) & $5320-12920$ \\
Father & 44 & 5659 (postoperative day 12) & $5320-12920$ \\
Brother & 9 & 9481 (postoperative day 12) & $5320-12920$ \\
\hline
\end{tabular}

oxygen partial pressure: $328 \mathrm{mmHg}$, bicarbonate concentration: $19.7 \mathrm{mmol} / \mathrm{L}$, base excess $-4.6 \mathrm{mmol} / \mathrm{L}$ ); therefore, we provided more hyperventilation to compensate for the mild respiratory acidosis. One hour and 30 minutes after the operation, the patient showed slight diaphragm and eyelid movement, and endotracheal tube suction induced slight movement, such as mild coughing. Two hours after the operation, having completely regained consciousness and showing adequate spontaneous ventilation, the patient was extubated and transferred to the recovery room.

As we suspected pseudocholinesterase deficiency after extubation, we examined the plasma pseudocholinesterase level in the patient and her family members on postoperative days 2 and 12. We confirmed pseudocholinesterase deficiency in the patient and her mother (Table 1).

Because prolonged apnea due to pseudocholinesterase deficiency is triggered after mivacurium or succinylcholine administration for muscle relaxation, pseudocholinesterase deficiency is clinically asymptomatic in patients without any previous history of receiving succinylcholine or mivacurium [2]. Acquired causes, including liver disease, renal disease, malnutrition, pregnancy, malignancy, burn, and cardiopulmonary bypass, can reduce pseudocholinesterase levels [1,3]. Several drugs, including anticholinesterase, contraceptives, monoamine oxidase inhibitors, pancuronium, oral bronchodilators, and metoclopramide, also can cause decreased pseudocholinesterase levels [1,4].

Although sufficient pyridostigmine was administered to reverse muscle relaxation in the patient, she showed persistent apnea and did not show any response to external stimuli. In such a situation, a reasonable approach is to examine the patient's response to peripheral nerve stimulation; in reality, however, many private clinics in Korea lack a peripheral nerve stimulator to assess neuromuscular blockade. Despite the additional administration of pyridostgmine, the patient did not exhibit adequate spontaneous respiration until 2 hours after the operation. Thus, we suspected prolonged apnea due to pseudocholinesterase deficiency. Our patient and her family members had no previous experience of receiving muscle relaxants, and genetically transmitted pseudocholinesterase deficiency was suspected. Therefore, it was important to determine the pseudocholinesterase levels in the patient and all family members [4]. Because pseudocholinesterase deficiency is transmitted in an autosomal recessive pattern and the frequency of apnea from a genetic abnormality of pseudocholinesterase is between $1: 480$ and $1: 3200$, we believe that the pseudocholinesterase deficiency observed in the patient and her mother has an inherited cause [4,5]. Although the dibucaine, fluoride, chloride, and urea number test can identify the genetic variation of pseudocholinesterase deficiency, we were unable to perform it on this patient; to the best of our knowledge, this kind of test is not commercially available in Korea. We rechecked the pseudocholinesterase levels in the patient and checked the pseudocholinesterase levels in her family members 12 days after the surgery. As we confirmed low pseudocholinesterase levels in the patient and her mother, we think that this pseudocholinesterase deficiency is associated with a genetically inherited cause rather than an iatrogenic cause, such as the additional pyridostigmine administration. Taking this case into consideration, after succinylcholine administration, anesthesiologists should apply a peripheral nerve stimulator to assess the extent of muscle relaxation reversal or carefully observe the return of spontaneous respiration, and then determine whether to administer an additional non-depolarizing muscle relaxant.

In conclusion, if pseudocholinesterase deficiency is suspected of causing unexpected prolonged apnea after succinylcholine administration, the following measures are very important: 1) controlled mechanical ventilation with sufficient sedation until succinylcholine is completely metabolized; 2) determination of pseudocholinesterase levels in the patient and all family members; 3 ) avoidance of further exposure to succinylcholine, mivacurium, and aminoester local anesthetics; and 4) notification given to medical doctors on any subsequent hospital visit that a patient has pseudocholinesterase deficiency.

\section{References}

1. Leadingham CL. A case of pseudocholinesterase deficiency in the PACU. J Perianesth Nurs 2007; 22: 265-71.

2. Kendrick K. Prolonged paralysis related to mivacurium: a case study. J Perianesth Nurs 2005; 20: 7-12.

3. Park SJ, Baik HJ, Kim KS, Cho DS. Comparison of pseudocholinesterase activity between nonpregnant and term-pregnant women with the genotypically normal ezyme. Korean J Anesthesiol 1996; 31: 613-8.

4. Soliday FK, Conley YP, Henker R. Pseudocholinesterase deficiency: a comprehensive review of genetic, acquired, and drug influences. AANA J 2010; 78: 313-20.

5. Naguib M, Lien CA. Pharmacology of muscle relaxants and their antagonists. In: Miller's anesthesia. 7th ed. Edited by Miller RD, Eriksson LI, Wiener-Kronish JP, Young WL: Philadelphia, Churchill Livingstone/Elsevier. 2010, pp 862-4. 